\title{
Abstracts From the Wilderness Medical Society's 2003 Winter Scientific Meeting
}

\author{
The Primalquest for Air: A Survey of Acute Mountain \\ Sickness in Expedition Adventure Racers
}

Background.-Adventure racing is one of the fastest growing outdoor sports in the United States and the world. Expedition level races vary from 3 to 10 days in duration, occurring in the most extreme of outdoor terrain and conditions. The race is a team event, and all members must complete the entire course. The Primalquest adventure race in July 2002 began at a base of over 9000 feet, ascended to over 13000 feet, and involved over 40000 feet of elevation gain in the first 12 hours of the race. Medication use is restricted, and no racer is allowed the use of conventional prophylactic medications for altitude sickness.

Objective.- - To determine the incidence of acute mountain sickness (AMS) requiring medical treatment or race withdrawal and other altitude-related disorders occurring during an expedition adventure race.

Methods.-All racers underwent a mandatory prerace medical assessment. All racers were asked to fill out an Environmental Symptoms Questionnaire version 3-R (ESQ-3R) during the medical check-in 24 hours prior to the race. An on-site medical team, trained in the recognition of acute-altitude illness, provided medical treatment for all racers. A standard medical encounter form was used in all patient encounters. Data on illness incidence were recorded in medical treatment logs and abstracted from the logs after race completion.

Results.- Sixty-two coed teams of 4 were entered into the race. Two hundred forty-five of 248 racers completed the prerace ESQ-3R. Thirty-six teams completed the race. Preliminary analysis of the data indicates an overall incidence of altitude illness requiring medical treatment of $12 \%$, with AMS accounting for $11.3 \%$ of the cases. Two racers evacuated for suspected highaltitude pulmonary edema (HAPE) account for the difference. Altitude illness resulted in $14.3 \%$ of the medical withdrawals from the race. An additional 50\% of the teams reported symptoms consistent with altitude illness but did not require medical intervention or result in withdrawal from the race.

Conclusion.-Expedition adventure racing continues to push the frontiers of human endurance in nature's most extreme environments. Altitude illness requiring medical treatment appears to have a significant impact during these events with an overall incidence of $12 \%$. Altitude illness accounted for $14.3 \%$ of the medical withdrawal from the event. Further analysis of the data is ongoing to determine the correlation between the racer's home altitude, prior altitude illness, and factors affecting successful completion of the event. Further research is needed to determine the factors that contribute to the development of altitude illness during these events and suggest avenues to reduce or modify its impact on the racer.

\author{
Timothy S. Talbot, MD \\ David Townes, MD, MPH, FACEP \\ Ian S. Wedmore, MD, FACEP \\ Fort Lewis, WA, USA
}

The Efficacy of Low-Dose Acetazolamide for Acute Mountain Sickness Prophylaxis

Objectives. - The minimum effective dose of acetazolamide for the prophylaxis of acute mountain sickness (AMS) has yet to be elucidated. The purpose of this study was to investigate the efficacy of acetazolamide at a dose of $125 \mathrm{mg}$ twice daily for AMS prophylaxis.

Methods.-Two hundred hikers (100 treatment subjects and 100 controls) were recruited at 3540-m elevation for this openlabel study as they ascended to a peak elevation of $5416 \mathrm{~m}$. Treatment subjects took acetazolamide $125 \mathrm{mg}$ orally twice a day, starting the night before they continued their ascent. Controls were asked to take nothing. A questionnaire was used to collect symptom data. AMS was defined per the Lake Louise criteria.

Results. - The final group sizes for analysis were 67 (29 females, 38 males) in the treatment group and 69 (30 females, 39 males) in the control group. The groups were comparable with respect to age, gender, and pulse oximetry at the time of recruitment. There was no significant difference in the rates of AMS between the 2 groups overall or among the men alone. For women, however, $63 \%$ of those in the control group developed AMS vs $34 \%$ of those in the treatment group. This represented a significant difference $(P<.05)$.

Conclusions. - The demonstrated gender-based discrepancy was most likely due to a dose-weight relationship. Although the studied dose was effective in women, this study demonstrates that $125 \mathrm{mg}$ of acetazolamide twice daily is not effective for AMS prophylaxis in all-comers. Further study of weight-based doses would be useful in order to determine a minimum prophylactic dose effective in both genders.

Chris C. Hoag, BSc Janet Berkman, MSc Andre R. Dabrowski, PhD Ottawa, Ontario, Canada

Virginia W. Robinson, BSc, MD, CCFP(EM) Kitchener, Ontario, Canada

Buddha Basnyat, MD Kathmandu, Nepal 
Risk Factors and Laboratory Trends in Acute Mountain Sickness

Introduction.-The pathophysiology of acute mountain sickness (AMS) is poorly understood. In particular, the role of dehydration is not clear. Moreover, there is little research in native populations at moderate altitude. Every August, thousands of Nepalis ascend from 2050 to $4500 \mathrm{~m}$ in 1 to 3 days for a religious pilgrimage.

Methods.-This was a prospective study with 2 parts. The first part was a cohort study of 38 Nepali pilgrims with moderate-altitude exposure to determine the physiologic changes that occur at altitude. Adult volunteers were initially enrolled at low altitude $(2050 \mathrm{~m})$. The following data were collected: age, gender, vital signs, AMS symptoms, and laboratory data, including hematocrit, Chem-7, and venous blood gas (VBG). Blood was analyzed using an i-Stat machine. When these subjects reached the high-altitude site, the same data were collected as well as rate of ascent and water consumption. The second part was a cross-sectional study of people at the high-altitude site to determine the physiologic variables that are associated with AMS. Identical data as listed above were collected.

Results. - In the first part of the study, data were obtained on 38 patients at both low and high altitudes. The following variables were significantly different at high altitude within these patients $(P<.05)$ : serum urea nitrogen/creatinine, heart rate, respiratory rate, hematocrit, $\mathrm{pH}$, and Lake Louise score all increased; bicarbonate, creatinine, oxygen saturation, and temperature decreased. Four patients could not be evaluated for AMS development. Of the 34 remaining patients, 16 (47\%) were diagnosed with AMS; these patients had a significantly smaller increase in their hematocrit $(0.20$ vs 3.11$)$ and a smaller decrease in body temperature $(-0.16$ vs -0.71$)$ and lower water consumption ( 1.3 vs $2.4 \mathrm{~L})$ than the patients who did not develop AMS. In the second part of the study, 96 subjects were enrolled. Of these, 41 patients (43\%) were diagnosed with AMS. These patients had lower water consumption (1.8 vs $3.1 \mathrm{~L})$ and oxygen saturation $(80.0 \%$ vs $82.8 \%)$ and higher heart rate $(102.5$ vs 95.5$)$, body temperature $\left(36.4^{\circ} \mathrm{C}\right.$ vs $\left.36.0^{\circ} \mathrm{C}\right)$, and serum chloride (108.0 vs 106.6$)$ than their nonAMS counterparts.

Conclusion.-This is the largest study to date of subjects exposed to high altitude, during which extensive laboratory data were collected onsite. We are unable to make a clear association between dehydration and the development of AMS. However, decreased water consumption was linked to the development of AMS. Other vital signs and laboratory trends indicate a relationship between dehydration and AMS. Larger controlled studies are needed to better delineate a causal relationship.

Lisa A. Rabinowitz Heemun K. Kwack Mark B. Shah Thomas A. Cumbo Darren A. Braude Cameron S. Crandall Buddha Basnyat Albuquerque, NM, USA

\section{Clinical Presentation of Exercise-Induced Hyponatremia}

Objective.-Exercise-induced hyponatremia is a life-threatening process that has frequently been misdiagnosed due to lack of differentiation from other heat-related illnesses. Most recent studies indicate that exertional hyponatremia is secondary to dilutional effects of over-hydration in otherwise healthy individuals. This study set out to describe the signs and symptoms of exercise-induced hyponatremia.

Methods. - As Grand Canyon National Park sees a preponderance of exertional hyponatremia during summer months, a retrospective chart review of Emergency Services (EMS) calls during the summers of 2000-2002 was performed. Point of care sodium measurements were performed at rangers' discretion. Patients with recorded sodium below $135 \mathrm{mEq} / \mathrm{L}$ were deemed eligible for study enrollment. Symptoms and vital signs were extracted from prehospital records.

Results.- Thirty-two cases of acute hyponatremia were identified. The median age was 51 (range 17-63). A female predominance was noted, with a female:male ratio of $3: 1$. Mean serum sodium was 126 (range 111-134). The most common symptoms identified were nausea $(84 \%)$, mental status changes $(67 \%)$, agitation/anxiety $(67 \%)$, fatigue $(59 \%)$, vomiting $(47 \%)$, dizziness $(47 \%)$, and tremors $(47 \%)$. Less commonly noted symptoms included headaches $(31 \%)$, lack of muscle coordination (25\%), diarrhea (19\%), incontinence (13\%), leg cramps (9\%), and grand mal seizures (9\%). The 3 most common symptoms in men were mental status changes, dizziness, and nausea. Women most commonly presented with agitation, weakness, and nausea. Vital signs on initial presentation were also analyzed. The median temperature was 97.8 (range 91.5-98.8); the median pulse was 80 (range 48-120); the median systolic blood pressure was 128 (range 101-180); the median pulse pressure was 50 (range 22-80); and the median respiration rate was 13 (range 10-26). Average recorded fluid intake was 6.5 liters.

Conclusions. - This study found exercise-associated hyponatremia was more common in women than in men. The most common symptoms associated with exertional hyponatremia were nausea, mental status changes, agitation/anxiety, fatigue, vomiting, dizziness, and tremors. Analysis of vital signs revealed that tachycardia, hypotension, and hyperthermia, frequently seen in other heat-related illnesses, were absent in those suffering from hyponatremia. However, hyponatremic patients did have greater than normal pulse pressures, likely secondary to hyperhydration. It is hoped that a better understanding of the symptom complex associated with hyponatremia, plus attention to vital signs, should effectively differentiate this disease process from other heat-related illnesses.

Hollynn Larrabee, MD

Steven Stephanides, MD

Cincinnati, OH, USA

Sherrie Collins, EMT-P

Grand Canyon, AZ, USA 


\section{Energy Balance and Change in Substrate Utilization During Extended Cold Weather Operations}

Training enhances fat utilization during moderate exercise. We tested if a $400-\mathrm{km}$ winter expedition would evoke a shift toward fat utilization. Pre- and postexpedition, enriched $\left(\mathrm{U}^{13} \mathrm{C}\right)$ glucose was ingested during a 2-h exercise protocol (workload similar to the trek) (5 males). This protocol determined contributions of endogenous and exogenous glucose (EndGluc and ExGluc) and fat to energy yield. Isotope clearance $\left({ }^{2} \mathrm{H}_{2}{ }^{18} 0\right)$ was used to estimate daily energy expenditure (EE) $(n=3)$. Subjects were $( \pm \mathrm{SD}) 36 \pm 4$ years old and $180.2 \pm 6 \mathrm{~cm}$ tall. The 19-day trek decreased body mass $(88.8 \pm 12$ to 81.8 $\pm 12 \mathrm{~kg})$ and body fat $(19.1 \% \pm 3 \%$ to $16.6 \% \pm 3 \%)$. Distance increased from $18.8 \mathrm{~km} / \mathrm{d}$ (days $1-10$ ) to $23.1 \mathrm{~km} / \mathrm{d}$ (days 11-19). EE increased from $25.6 \pm$ to $28.9 \pm \mathrm{MJ} / \mathrm{d}$. The trek had no pre-to-post effect on percentage of total energy contribution from fat $(21.5 \% \pm 4 \%$ to $22.3 \% \pm 4 \%)$, ExGluc $(22.4 \% \pm 5 \%$ to $23.6 \% \pm 5 \%)$ or EndGluc $(39.4 \% \pm 9 \%$ to $38.5 \% \pm 8 \%$ ) during the 2 -hour test. However, from the start to the finish of each test, an increased contribution of ExGluc $(18.1 \%-37.6 \%)$ spared use of EndGluc (54.8\%-43.7\%) and fat (27.1\%-18.6\%). At lower workloads, frequent ingestion of glucose may effectively spare endogenous energy stores. Support Natural Science and Engineering Research Council (NSERC) Canada.

Gordon G. Giesbrecht, PhD

Claudia Marrao, MSc

Pritphal Pachu, MSc Michael Campbell, $\mathrm{PhD}$

Winnepeg, Manitoba, Canada

Francois Perronet, $\mathrm{PhD}$

Hanen M'Kaouar, MSc

Montreal, Quebec, Canada

Klaas Westerterp, PhD Maastricht, Netherlands 